\title{
The Use of MicroRNAs for the Early Diagnosis of Dementia in Patients Affected by Hearing Loss
}

\author{
Cha Dong Yeo (iD · Moo Kyun Park (i) \\ Department of Otorhinolaryngology-Head and Neck Surgery, Seoul National University Hospital, Seoul National University College of Medicine,
} Seoul, Korea

Dementia is one of the greatest public health challenges facing elderly people worldwide. It has been predicted that by 2050 , the number of patients with dementia worldwide will increase approximately three times, from 46.8 million to 113.5 million. Alzheimer disease makes up more than $60 \%$ of dementia cases [1]. Hearing loss is highly prevalent in the elderly and is severely undertreated. Around two-thirds of people older than 70 years are affected by hearing loss, but fewer than $20 \%$ of adults with hearing loss receive care (e.g., hearing aids). As the global population is growing and aging, the number of people with hearing loss is increasing rapidly. In fact, the World Health Organization [2] is currently predicting a related increase in the incidence of hearing loss around the world. Globally, there are about 466 million people with debilitating hearing loss; this figure is expected to grow to 630 million by 2030 and over 900 million by 2050 unless action is taken. Untreated hearing loss also imposes a significant economic burden, with an estimated annual global cost of $\$ 750$ billion. Hearing loss has only recently been identified as a possible risk factor for dementia and has not been prioritized in the care of patients with or at risk of dementia. However, the latest prospective cohort studies have shown that hearing loss in individuals who are cognitively healthy, but hearing-impaired at baseline, is an independent risk factor of incident dementia (i.e., new cases of dementia) [3]. Therefore, methods for the early diagnosis and rehabilitation of hearing loss are needed to prevent dementia.

MicroRNAs (miRNAs) are 20- to 22-nucleotide single-stranded non-coding RNAs that bind to the 3 '-untranslated regions of mRNA to degrade the genetic code of the mRNA itself or to repress translation. miRNAs are involved in cell death, differentiation, and proliferation, and miRNA expression abnormalities are known to play an important role in cancer, cardiovascular, and autoimmune diseases, hearing loss, and neural development and plasticity. Approximately $70 \%$ of the currently known miRNAs are expressed in the brain. miRNAs may also play a role in neu- rodegeneration, and specific miRNAs may be associated with neurodegenerative diseases [4]. At present, the study of miRNAs represents one of the most active areas of genomics and has shown promising outcomes.

Given the relationship between hearing loss and dementia, the identification of miRNAs associated with cognitive impairment after hearing loss could facilitate the early diagnosis of Alzheimer disease in people affected by hearing loss. Not many studies have used miRNAs to diagnose cognitive impairment in patients with hearing loss. Recently, Mun et al. [5] reported an interesting study on miRNAs in animal models of hearing loss. They suggested that miRNAs can be used to diagnose cognitive impairment after hearing loss. In addition, they reported that miR-376a-3p and miR-598-3p were related to cognitive impairment after hearing loss [5]. Further research is needed to determine whether miRNAs in human cerebrospinal fluid and/or blood can be assessed and to identify the genes controlled by these miRNAs. Research on changes in miRNA levels in patients with hearing loss and dementia will contribute to the early diagnosis of dementia in patients with hearing loss and possibly to the prevention of cognitive impairment through hearing rehabilitation. In addition, these results could provide a new treatment approach for dementia and hearing loss.

\section{CONFLICT OF INTEREST}

No potential conflict of interest relevant to this article was reported.

\section{ORCID}

Cha Dong Yeo https://orcid.org/0000-0002-5802-6444

Moo Kyun Park https://orcid.org/0000-0002-3621-4524

Copyright (C) 2021 by Korean Society of Otorhinolaryngology-Head and Neck Surgery.

This is an open-access article distributed under the terms of the Creative Commons Attribution Non-Commercial License (https://creativecommons.org/licenses/by-nc/4.0)

which permits unrestricted non-commercial use, distribution, and reproduction in any medium, provided the original work is properly cited. 


\section{AUTHOR CONTRIBUTIONS}

Conceptualization: MKP. Data curation, Formal analysis, \& Methodology: CDY. Project administration \& Visualization: MKP. Writing-original draft: CDY.Writing-review \& editing: MKP.

\section{REFERENCES}

1. Larsson SC, Markus HS. Does treating vascular risk factors prevent dementia and alzheimer's disease? A systematic review and metaanalysis. J Alzheimers Dis. 2018;64(2):657-68.

2. World Health Organization. Addressing the rising prevalence of hearing loss. [Internet]. Geneva: World Health Organization; 2018 [cited 2021 Jan 20].Available from: http://apps.who.int/iris/bitstream/ handle/10665/260336/9789241550260-eng.pdf;jsessionid=420589 F88F058B91C3A8B93AF67587C2? sequence $=1$.

3. Deal JA, Betz J, Yaffe K, Harris T, Purchase-Helzner E, Satterfield S, et al. Hearing impairment and incident dementia and cognitive decline in older adults: the health ABC study. J Gerontol A Biol Sci Med Sci. 2017 May;72(5):703-9.

4. Wei CW, Luo T, Zou SS, Wu AS. Research progress on the roles of microRNAs in governing synaptic plasticity, learning and memory. Life Sci. 2017 Nov;188:118-22.

5. Mun SK, Chae H, Piao XY, Lee HJ, Kim YK, Oh SH, et al. MicroRNAs related to cognitive impairment after hearing loss. Clin Exp Otorhinolaryngol. 2021 Feb;14(1):76-81.

Received January 5, 2021

Accepted January 5, 2021 